\title{
Interventional closure of an unusual broncho-pleural fistula with superglue
}

\section{To the Editor}

Rheumatoid arthritis is a multisystemic disorder which has multiple extraarticular manifestations. One of the most common pulmonary manifestations is in the form of pulmonary nodules [1]. These patients are at higher risk of recurrent pneumothorax which can get complicated in form of broncho/alveolo- pleural fistula [2]. This can cause significant increase in morbidity. Here we report a case of recurrent pneumothorax who was diagnosed to have rheumatoid arthritis with multiple pulmonary nodules and the broncho-pleural fistula. She was successfully managed with bronchoscopic intrabronchial cyanoacrylate glue instillation.

A 42-year-old female presented to another hospital with history of progressive dyspnoea and right sided pleuritic chest pain of 4 days duration. On evaluation her chest radiograph revealed right sided pneumothorax. She was managed with intercostal drain (ICD) insertion and conservative management. ICD was removed after 7 days as her pneumothorax resolved and there was no evidence of air-leak and she was discharged. Unfortunately, patient reported again after 10 days with similar complaints and further evaluation revealed a recurrent pneumothorax (Figure 1A). ICD reinsertion was done and a persistent air-leak (Cerfolio grade-2) was also identified. During her hospital stay, she complained of pain and progressive swelling of joints of her hands and legs, especially the small joints. On evaluation, she was found to have raised rheumatoid factor levels and anti- cyclic citrullinated protein (anti-CCP) antibody and was diagnosed to have rheumatoid arthritis and was commenced on disease modifying anti rheumatic drugs. Her contrast enhanced computed tomography of chest showed numerous calcified nodules in bilateral lungs with patchy bronchiectasis and coarse reticulations (Figure 1C). There were features of mosaic attenuation with patchy areas of air-trapping seen in bilateral lungs. She underwent bronchoscopy with $6.0 \mathrm{~mm}$ diameter flexible video- bronchoscope $(2.8 \mathrm{~mm}$ channel, Olympus BF-1T150, Olympus Corporation, Japan) which showed unhealthy mucosa in right lower lobe (RLL) bronchus which bled on touch. Broncho-pleural fistula (BPF) was localised by sequential occlusion with balloon occlusion catheter (Fogarty; Edwards Lifesciences, Irvine, CA, USA) to lateral basal segment of RLL. We decided to close the BPF with cyano acrylate glue. N-butyl cyano acrylate glue was filled in $2 \mathrm{~mL}$ syringe under strict dry condition. We used transbronchial needle aspiration needle (21G, $15 \mathrm{~mm}$ in length, Olympus Corporation, Japan) for delivery of the glue which is otherwise used for diagnostic aspiration from mediastinal lymph nodes. It was inserted through the working channel of the bronchoscope and the glue was instilled under direct observation to the site of leak. We kept the bronchoscope about two centimetres away from the catheter tip to prevent damage to the bronchoscope and we also used additional precaution in form of Fogarty balloon which was inflated between the tip of catheter and the distal tip of scope to prevent any spill of the glue on the scope (Figure 1D). The procedure was successful and her right lung showed radiologic expansion with resolution of the leak and chest tube was 


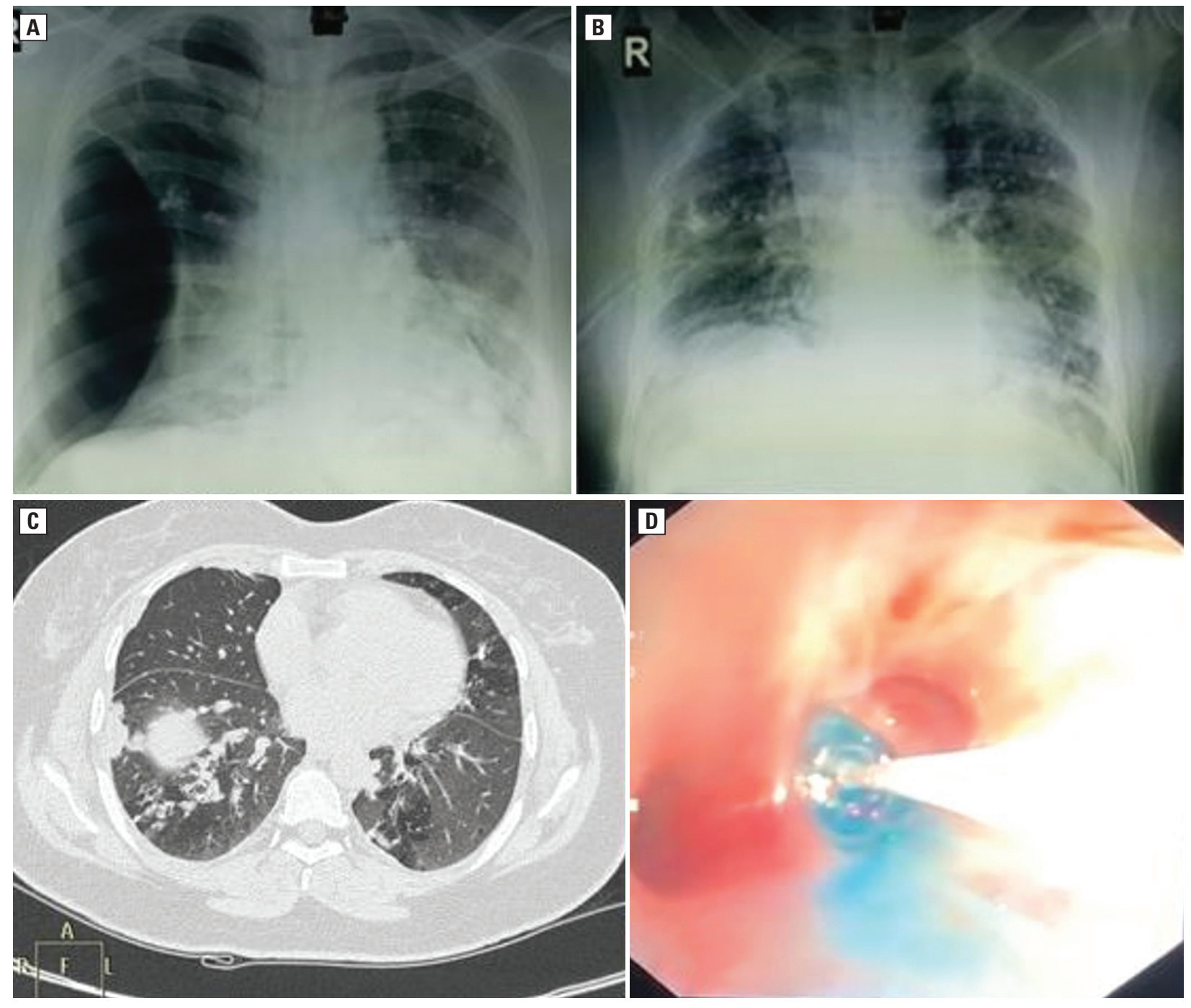

Figure 1A. Chest Skiagram showing right sided pneumothorax; B. Chest skiagram showing resolved pneumothorax post intrabronchial instillation of cyano-acrylate glue; C. Computed tomography of chest showing bilateral rheumatoid nodules; D. Fibreoptic bronchoscopic picture showing intrabronchial glue installation

removed 3 days after the procedure (Figure 1B). She is presently asymptomatic.

Rheumatoid arthritis is a systemic disease which causes progressive and symmetrical inflammatory destruction of joints due to antibodies [1]. It also has extra-articular manifestations in form subcutaneous nodules, vasculitis, eye involvement and pulmonary disease [2]. The pulmonary involvement of RA constitutes of pleural effusion, pneumothorax, pulmonary nodules, pulmonary artery hypertension, bronchiolitis, organising pneumonia and Caplans syndrome [2, 3] The pulmonary necrobiotic nodules can cause haemoptysis, pneumothorax and bronchopleural fistula. These patients are prone for recurrent pneumothorax and bronchopleural fistula which can cause significant morbid- ity and prolong hospital stay with increased chances of infection [4,5] The management of bronchopleural fistula is a challenging task and includes surgical and endobronchial interventions. The surgical options include chronic open drainage, direct stump closure with intercostal muscle reinforcement, omental flap, trans-sternal bronchial closure, and thoracoplasty with or without extrathoracic chest wall muscle transposition [6-8]. Video assisted bronchoscopy can be both diagnostic and therapeutic in these patients as it can be used to localize the leak and allows introduction of sealants. The advantages of bronchoscopic procedure are cost effectiveness, quick action, lesser hospital stay and can also be done on out-patient basis [6-8]. The important factor which determines 
success of this method is the location and size of air-leak. The success rate is higher in peripheral and alveolo-pleural fistulas less than $4 \mathrm{~mm}$ [7]. The leak should be directly visualised and there should be reduction or stoppage of leak on occlusion with endobronchial catheter tip [6, 8]. Video bronchoscopic guided endobronchial glue installation is effective non- operative method of closure of alveolo-pleural fistula.

\section{Conflict of interest}

None declared.

\section{References:}

1. Shaw M, Collins BF, Ho LA, et al. Rheumatoid arthritis-associated lung disease. Eur Respir Rev. 2015; 24(135): 1-16, doi: 10.1183/09059180.00008014, indexed in Pubmed: 25726549.

2. Crisp AJ, Armstrong RD, Grahame R, et al. Rheumatoid lung disease, pneumothorax, and eosinophilia. Ann Rheum Dis. 1982; 41(2): 137-140, doi: 10.1136/ard.41.2.137, indexed in Pubmed: 7073341.
3. Corcoran JP, Ahmad M, Mukherjee R, et al. Pleuro-pulmonary complications of rheumatoid arthritis. Respir Care. 2014; 59(4): e55-e59, doi: 10.4187/respcare.02597, indexed in Pubmed: 23962501.

4. Alfara JJF, Sebastián GG, González CL. Recurrent pneumothorax due to rheumatoid arthritis. Archivos de Bronconeumología (English Edition). 2004; 40(10): 476, doi: 10.1016/ s1579-2129(06)60359-5.

5. Saravana S, Gillott T, Abourawi F, et al. Spontaneous pneumothorax: an unusual presentation of rheumatoid arthritis. Rheumatology (Oxford). 2003; 42(11): 1415-1416, doi: 10.1093/ $\underline{\text { rheumatology/keg344, indexed in Pubmed: } 14578435 .}$

6. Katoch CDS, Chandran VM, Bhattacharyya D, et al. Closure of bronchopleural fistula by interventional bronchoscopy using sealants and endobronchial devices. Med J Armed Forces India. 2013; 69(4): 326-329, doi: 10.1016/j.mjafi.2013.04.009, indexed in Pubmed: 24600137.

7. Chawla RK, Madan A, Bhardwaj PK, et al. Bronchoscopic management of bronchopleural fistula with intrabronchial instillation of glue (N-butyl cyanoacrylate). Lung India. 2012; 29(1): 11-14, doi: 10.4103/0970-2113.92350, indexed in Pubmed: $\underline{22345907 .}$.

8. Marwah V, Katoch CDS, Kumar K, et al. Bronchoscopic device closure of postoperative bronchopleural fistulae: Novel devices and innovative techniques. Lung India. 2020; 37(2): 107-113, doi: 10.4103/lungindia.lungindia_179_19, indexed in Pubmed: $\underline{32108593}$. 